\title{
THE SPACE OF HOMEOMORPHISMS ON A COMPACT TWO-MANIFOLD IS AN ABSOLUTE NEIGHBORHOOD RETRACT
}

\author{
BY \\ R. LUKE AND W. K. MASON( $\left.{ }^{1}\right)$
}

Abstract. The theorem mentioned in the title is proved.

1. Introduction. Throughout this paper $M^{n}$ will denote a compact, metric, $n$-manifold, $n \geqq 1$. If $M^{n}$ is without boundary $H\left(M^{n}\right)$ will denote the space (under the sup norm topology) of all homeomorphisms of $M^{n}$ onto $M^{n}$. If $M^{n}$ has nonempty boundary $H\left(M^{n}\right)$ will denote the space of all homeomorphisms of $M^{n}$ onto $M^{n}$ which leave the boundary pointwise fixed.

A result which has triggered a great deal of work recently is the following. The space of all orientation preserving homeomorphisms of the unit interval $[0,1]$ onto itself is homeomorphic to $l_{2}$, the separable hilbert space of square summable sequences [2]. A natural question is whether $H\left(M^{n}\right)$ is locally homeomorphic to $l_{2}$ for every $M^{n}[13$, p. 792], [24, Problem M1].

It is well known that $H\left(M^{n}\right)$ is a complete separable metric space [6, p. 265]. Mason [17] has shown that if $K$ is a sigma-compact subset of $H\left(M^{n}\right)$, then $H\left(M^{n}\right)-K$ is homeomorphic to $H\left(M^{n}\right)$. Geoghegan [8] has shown that $H\left(M^{n}\right) \times l_{2}$ is homeomorphic to $H\left(M^{n}\right)$ (for a generalization see Keesling [14]). Černavskiǐ [4] and Edwards and Kirby [7] have shown that $H\left(M^{n}\right)$ is locally contractible, (earlier, Hamstrom and Dyer [10] showed that $H\left(M^{2}\right)$ was locally contractible).

The homotopy groups of $H\left(M^{2}\right)$ have been studied by Hamstrom [9] and McCarty [16] (see also Morton [19]). Mason [18] showed that if $D$ is a 2-cell then $H(D)$ is an absolute retract (a problem originally raised by E. Michael, see [26, p. 229]).

In this paper we show that $H\left(M^{2}\right)$ is an absolute neighborhood retract (Theorem 18). During the course of the proof we show that various other function spaces are absolute neighborhood retracts. Some of these are: the space of embeddings of a 2-cell $D$ into the plane $E^{2}$, the space of embeddings of $\mathrm{Bd}(D)$ into $E^{2}$, and the space of all embeddings of $D$ into $E^{2}$ which are holomorphic on Int $(D)$ (see $\S 4$ ).

Received by the editors February 5, 1971.

AMS 1970 subject classifications. Primary 57A05, 58D10.

Key words and phrases. Retract, absolute neighborhood retract, two-manifold, twomanifold function space, space of homeomorphisms, infinite-dimensional manifold.

(') Research partially supported by NSF GP-20861.

Copyright (C) 1972, American Mathernatical Society 
The basic idea of the proof is to use the result that $H(D)$ is an absolute retract, decompose $M^{2}$ into 2-cells, and use the techniques of Hamstrom and Dyer [10] on the decomposition.

All function spaces mentioned in this paper will be topologized by the "sup norm" distance function (see $\S 2$ ).

2. Definitions and notation. The statement that a metric space $X$ is an absolute neighborhood retract (ANR) means that whenever $X$ is embedded as a closed subset $Z_{0}$ of a metric space $Z$, there is a retraction of an open neighborhood of $Z_{0}$ onto $Z_{0}$.

A sequence $X_{1}, X_{2}, \ldots$ converges 0 -regularly to a set $X_{0}$ if $X_{1}, X_{2}, \ldots$ converges to $X_{0}$, and for every $\varepsilon>0$, a $\delta>0$ and an integer $N>0$ exist such that, if $n>N$, any two points $x, y$ of $X_{n}$, with dist $(x, y)<\delta$, lie together in a connected subset of $X_{n}$ of diameter less than $\varepsilon$.

By map we mean continuous function. $f: A \rightarrow B$ means that the function $f$ takes $A$ onto $B$. If $A$ and $B$ are metric spaces, with $A$ compact, and $f_{1}, f_{2}: A \rightarrow B$ are maps, then dist $\left(f_{1}, f_{2}\right)$, the distance between $f_{1}$ and $f_{2}$, will be

$$
\sup _{x \in A} \operatorname{dist}\left(f_{1}(x), f_{2}(x)\right) \text {. }
$$

If $M$ is a manifold, $\mathrm{Bd}(M)$ denotes the boundary of $M$, and Int $(M)$ denotes the interior of $M$.

$E^{2}$ denotes the Euclidean plane, and $\mathscr{C}^{1}$ the complex number plane. By 1 we shall often mean the point $1+0 i \in \mathscr{C}^{1}$.

If $X \subset \mathscr{C}^{1}$ is a point set and $f: X \rightarrow \mathscr{C}^{1}$ is a map, then $f$ is holomorphic at a point $x \in X$ if $f$ has a (complex) derivative, $f^{\prime}(z)$, for all $z$ in some neighborhood (in $\mathscr{C}^{1}$ ) of $x$. If $f$ is $1-1, f$ is orientation preserving on $X$, if given any simple closed curve $J$ in $X$ and a positive transversal $\alpha$ of $J$, then $f \circ \alpha$ is a positive transversal of $f(J)$ (see [23, Chapter 5, §2]).

An $\operatorname{arc} B$ is a spanning $\operatorname{arc}$ of a disk $Z$ if $B \subset Z$ and $B \cap \operatorname{Bd}(Z)$ are the endpoints of $B$.

If $A$ and $B$ are point sets, $A+B$ denotes the union (sum) of $A$ and $B .\left\{A_{n}\right\} \rightarrow A$ means that the sequence $A_{0}, A_{1}, \ldots$ converges to $A$.

If $X \subset Z$ are manifolds in $E^{2}$ (or $\mathscr{C}^{1}$ ), then $E(X, Z)$ denotes the space of all embeddings of $X$ into $Z$ which are fixed on $X \cap \operatorname{Bd}(Z)$ (possibly $\operatorname{Bd}(Z)=\varnothing$ ), and which take $X \cap \operatorname{Int}(Z)$ into $\operatorname{Int}(Z) . E_{0}(X, Z)=\{f \in E(X, Z): f$ is orientation preserving\}. $A E(X, Z)=\{f \in E(X, Z): f$ is holomorphic on Int $(X)\}$.

3. Preliminaries on conformal mapping. It is a well-known result of complex analysis that if $D$ is the closed unit disk in $\mathscr{C}^{1}$, and $J$ is a simple closed curve in $\mathscr{C}^{1}$ bounding a closed (topological) disk $G$, then there is a homeomorphism $f$ of $D$ onto $G$ such that $f$ is holomorphic on Int $(D)$. Further, if $z_{0} \in \operatorname{Int}(G)$ and $z_{1} \in \operatorname{Bd}(G)$, there is a unique $f$ such that $f(0)=z_{0}$, and $f(1)=z_{1}[20$, Theorems 12.6, 14.8, 14.19].

The following continuity property of $\operatorname{such} f$ 's is not so well known. 
THEOREM 1. Suppose that for each nonnegative integer $i, J_{i}$ is a simple closed curve in $\mathscr{C}^{1}$ which bounds a closed (topological) disk $G_{i}$. Suppose

(1) $\left\{J_{i}\right\} \rightarrow J_{0} 0$-regularly,

(2) $z_{0} \in \operatorname{Int}\left(G_{i}\right)$ for some fixed $z_{0}, i=0,1,2, \ldots$,

(3) for each $i, i=0,1, \ldots, f_{i}: D \rightarrow G_{i}$ is a homeomorphism such that $f_{i}(0)=z_{0}$ and $f_{i}$ is holomorphic on Int $(D)$,

(4) $\left\{f_{2}(1)\right\} \rightarrow f_{0}(1)$.

Then $\left\{f_{i}\right\} \rightarrow f_{0}$ uniformly on $D$.

(Proof omitted.)

A version of this theorem is given in [10]. A proof may be dug out of [5, p. 191]. Perhaps the most elementary method of proof is to use Lindelöff's lemma [21, Chapter 3, §10] and mimic the nice proof of Lemma 12.1 in [21, Chapter 3, §12].

In order to state the next lemma we need the following notation. This notation will be used in $\S 4$ also. Suppose $f \in E_{0}\left(\operatorname{Bd}(D), E^{2}\right)$, where $D=\left\{z \in \mathscr{C}^{1}:|z| \leqq 1\right\}$ $=\left\{(x, y) \in E^{2}: x^{2}+y^{2} \leqq 1\right\}$. Let $A(f)$ be an annulus in $E^{2}$ such that $f(\operatorname{Bd}(D))$ $\subset$ Int $(A(f))$, and $f(\mathrm{Bd}(D))$ separates $\mathrm{Bd}(A(f))$. Let $x(f)$ be a point in the bounded component of $E^{2}-A(f)$. Define

$$
\begin{gathered}
w(A(f))=\left\{h \in E_{0}\left(\mathrm{Bd}(D), E^{2}\right): h(\mathrm{Bd}(D)) \subset \mathrm{Int}(A(f))\right. \text { and } \\
h(\mathrm{Bd}(D)) \text { separates } \mathrm{Bd}(A(f))\}, \\
V(A(f))=\left\{g \in A E\left(D, E^{2}\right): g(0)=x(f) \text { and } g \mid \mathrm{Bd}(D) \in w(A(f))\right\} .
\end{gathered}
$$

Theorem 1 and the remarks preceding Theorem 1 give us

LEMma 2. There is a map $\sigma^{\prime}: w(A(f)) \rightarrow V(A(f))$ such that $\sigma^{\prime}(h)(1)=h(1)$, and $\sigma^{\prime}(h)(\mathrm{Bd}(D))=h(\mathrm{Bd}(D))$, for all $h \in w(A(f))$.

LemMA 3 (SEE [10]). There is a map $\sigma: w(A(f)) \rightarrow E\left(D, E^{2}\right)$ such that $\sigma(h) \mid \operatorname{Bd}(D)$ $=h$, for all $h \in w(A(f))$.

Proof. For each $h \in w(A(f))$, let $h_{1}: \mathrm{Bd}(D) \rightarrow \mathrm{Bd}(D)$ be given by

$$
h_{1}=\left[\sigma^{\prime}(h)\right]^{-1} \circ h
$$

( $\sigma^{\prime}$ is the map of Lemma 2). Extend $h_{1}$ to a homeomorphism $H_{1}: D \rightarrow D$ by $H_{1}(r, \theta)=\left(r, \arg \left(h_{1}(1, \theta)\right)\right),(r, \theta)$ polar coordinates. Finally, let $\sigma(h)=\sigma^{\prime}(h) \circ H_{1}$. The continuity of $\sigma^{\prime}$ implies the continuity of $\sigma$.

Noting in the above proof that $h_{1}(1)=1$, we obtain

CoRollary 4. $w(A(f))$ is homeomorphic to $V(A(f)) \times \bar{H}(\mathrm{Bd}(D))$, where $\bar{H}(\mathrm{Bd}(D))$ is the space of all orientation preserving homeomorphisms of $\mathrm{Bd}(D)$ onto $\mathrm{Bd}(D)$ which are fixed at 1 .

Proof. If $h \in w(A(f))$, the mapping sending $h$ to $\left(\sigma^{\prime}(h),\left[\sigma^{\prime}(h)\right]^{-1} \circ h\right)$ is the required homeomorphism. 
Recall that if $Y$ is a spanning arc of the disk $D$ then $E(Y, D)$ is the space of all embeddings of $Y$ into $D$ which are fixed on the endpoints of $Y$ and which take $Y \cap \operatorname{Int}(D)$ into Int $(D)$. For the next lemma we think of $D$ as the set

$Y$ as the arc

$$
\left\{(x, y) \in E^{2}: 0 \leqq x \leqq 1,-1 \leqq y \leqq 1\right\},
$$

and define disks

$$
\left\{(x, y) \in E^{2}: 0 \leqq x \leqq 1, y=0\right\}
$$

$$
\begin{aligned}
& Z_{0}=\left\{(x, y) \in E^{2}: 0 \leqq x \leqq 1,-2 \leqq y \leqq 2\right\} \\
& Z_{1}=\left\{(x, y) \in E^{2}: 0 \leqq x \leqq 1,-2 \leqq y \leqq 0\right\} \\
& Z_{2}=\left\{(x, y) \in E^{2}: 0 \leqq x \leqq 1,0 \leqq y \leqq 2\right\}
\end{aligned}
$$

LEMMA 5. There are maps $\gamma: E(Y, D) \rightarrow E\left(Z_{1}, Z_{0}\right)$, and $\alpha: E(Y, D) \rightarrow E\left(Z_{2}, Z_{0}\right)$ such that $\alpha(f)|Y=\gamma(f)| Y=f, \quad \gamma(f) \mid \operatorname{Bd}\left(Z_{0}\right) \cap \operatorname{Bd}\left(Z_{1}\right)=\mathrm{Id}, \quad$ and $\alpha(f) \mid \operatorname{Bd}\left(Z_{0}\right)$ $\cap \operatorname{Bd}\left(Z_{2}\right)=\operatorname{Id}$, all $f \in E(Y, D)$.

Proof. Follows from Lemma 3.

Corollary 6. $E(Y, D) \times H\left(Z_{1}\right) \times H\left(Z_{2}\right)$ is homeomorphic to $\bar{H}\left(Z_{0}\right)$, where $\bar{H}\left(Z_{0}\right)=\left\{h \in H\left(Z_{0}\right): h \mid Y \in E(Y, D)\right\}$.

Proof. If $h \in \bar{H}\left(Z_{0}\right)$, the map sending $h$ to $\left(h \mid Y, h^{-1} \circ \gamma(h \mid Y), h^{-1} \circ \alpha(h \mid Y)\right)$ is the required homeomorphism.

\section{Some function spaces which are ANR's.}

Definition. Let $A\left(D, E^{2}\right)$ denote the space of all maps from $D=\left\{z \in \mathscr{C}^{1}:|z| \leqq 1\right\}$ into $E^{2}$ which are holomorphic on Int $(D)$.

LEMMA 7. $A\left(D, E^{2}\right)$ is an $A N R$.

Proof. $A\left(D, E^{2}\right)$ is a Banach space [20, Example 18.11], and hence an ANR [12, Chapter 3, Corollary 6.4].

Definitions. For $r$ a real number, $0<r<1$, let $D(r)=\left\{z \in \mathscr{C}^{1}:|z| \leqq r\right\}$. Let $A\left(D, E^{2}, r\right)=\left\{f \in A\left(D, E^{2}\right): f\right.$ is 1-1 on some neighborhood of $\left.D(r)\right\}$.

LEMMA 8. $A\left(D, E^{2}, r\right)$ is an $A N R, 0<r<1$.

Proof. We show that $A\left(D, E^{2}, r\right)$ is an open subset of $A\left(D, E^{2}\right)$. Suppose $f_{1}, f_{2}, \ldots$ is a sequence of elements of $A\left(D, E^{2}\right), f_{0}$ is an element of $A\left(D, E^{2}, r\right)$, and $\left\{f_{i}\right\} \rightarrow f_{0}$. For each $i, i=0,1,2, \ldots$, define $h_{i}$ : Int $(D) \times \operatorname{Int}(D) \rightarrow E^{2}$ by

$$
\begin{aligned}
h_{i}(x, y) & =\left(f_{i}(x)-f_{i}(y)\right) /(x-y) & & \text { if } x \neq y, \\
& =f_{i}^{\prime}(x) & & \text { if } x=y .
\end{aligned}
$$

For each $i, h_{i}$ is continuous on $\operatorname{Int}(D) \times \operatorname{Int}(D)[23$, p. 75]. Choose $\varepsilon>0$ so that $f_{0}$ is $1-1$ on $D(r+2 \varepsilon)$. Then $h_{0}(x, y) \neq 0$ for all $(x, y) \in D(r+\varepsilon) \times D(r+\varepsilon)$, since $f_{0}^{\prime}(x) \neq 0$ for all $x \in D(r+\varepsilon)$ [23, p. 84]. But $\left\{h_{i}\right\} \rightarrow h_{0}$ uniformly on $D(r+\varepsilon)$ $\times D(r+\varepsilon)$ (see proof of Theorem 7.3.1 in [23, p. 86]). Hence, for sufficiently large $i$, 
$h_{i}(x, y) \neq 0$, all $(x, y) \in D(r+\varepsilon) \times D(r+\varepsilon)$, and so $f_{i}(x) \neq f_{i}(y)$. Hence, for large $i$, $f_{i} \in A\left(D, E^{2}, r\right)$. It follows that $A\left(D, E^{2}, r\right)$ is open in $A\left(D, E^{2}\right)$. Therefore, by [12, Chapter 3, Proposition 7.9] and Lemma 7, $A\left(D, E^{2}, r\right)$ is an ANR.

LeMma 9. $\bigcap_{n=2}^{\infty} A\left(D, E^{2}, 1-1 / n\right)$ is an $A N R$.

Proof. We shall show that $\bigcap_{n=2}^{\infty} A\left(D, E^{2}, 1-1 / n\right)$ is a retract of $A\left(D, E^{2}, \frac{1}{2}\right)$. It will then follow that $\bigcap_{n=2}^{\infty} A\left(D, E^{2}, 1-1 / n\right)$ is an ANR [12, Chapter 3, Proposition 7.7]. Define $\lambda: A\left(D, E^{2}, \frac{1}{2}\right) \rightarrow\left(\frac{1}{2}, 1\right]$ by $\lambda(f)=\operatorname{Sup}\{r: f$ is $1-1$ on $D(r)\}$. It will be shown below that $\lambda$ is continuous. Define $R: A\left(D, E^{2}, \frac{1}{2}\right) \rightarrow \bigcap A\left(D, E^{2}, 1-1 / n\right)$ by $R(f)(z)=f(\lambda(f) \cdot z)$, all $z \in D \subset \mathscr{C}^{1}$, all $f \in A\left(D, E^{2}, \frac{1}{2}\right)$. Since $\lambda(f)=1$ for all $f \in \bigcap A\left(D, E^{2}, 1-1 / n\right)$, it follows that $R$ is a retraction, provided $\lambda$ is continuous.

Suppose $\lambda$ is not continuous. Suppose $\left\{f_{n}\right\} \rightarrow f_{0}$, but $\left\{\lambda\left(f_{n}\right)\right\} \rightarrow \lambda\left(f_{0}\right)$ for some sequence $f_{0}, f_{1}, \ldots$ of elements of $A\left(D, E^{2}, \frac{1}{2}\right)$.

Case 1. For infinitely many $n, \lambda\left(f_{0}\right)-\lambda\left(f_{n}\right)>\varepsilon$ for some $\varepsilon>0$. Choose $t$ so that $\lambda\left(f_{0}\right)-\varepsilon<t<\lambda\left(f_{0}\right)$. Then $f_{0} \mid D(t)$ is $1-1$. But then, as in the proof of Lemma 8 , $f_{n} \mid D(t)$ is $1-1$ for large $n$. This contradicts the assumption that $\lambda\left(f_{n}\right)<t$ for infinitely many $n$.

Case 2. For infinitely many $n, \lambda\left(f_{n}\right)-\lambda\left(f_{0}\right)>\varepsilon$ for some $\varepsilon>0$. Choose $t$ so that $\lambda\left(f_{0}\right)<t<\lambda\left(f_{0}\right)+\varepsilon$. Then for infinitely many $n, f_{n} \mid D(t)$ is $1-1$. But $\left\{f_{n}\right\} \rightarrow f_{0}$, so by a simple argument using Rouche's theorem (or see [21, p. 91]), $f_{0} \mid D(t)$ is $1-1$. This contradicts the assumption that $\lambda\left(f_{0}\right)<t$. The proof of Lemma 9 is complete.

For our next proof we need the following theorem of Hanner.

Theorem 10 (HANNer [11, Theorem 7.2]). A separable metric space $X$ is an $A N R$ provided there exist (1) a sequence of $A N R$ 's $Y_{1}, Y_{2}, \ldots$, (2) a sequence of maps $\phi_{i}: X \rightarrow Y_{i}, i=1,2, \ldots$, (3) a sequence of maps $\psi_{i}: Y_{i} \rightarrow X, i=1,2, \ldots$, and (4) a sequence of homotopies $H^{i}: X \times I \rightarrow X$ such that $H^{i}(x, 0)=x, H^{i}(x, 1)=\psi_{i} \phi_{i}(x)$, all $x \in X$, and $H^{1}, H^{2}, \ldots$ converges to the identity mapping $X \rightarrow X$.

Definition. $H^{1}, H^{2}, \ldots$ converges to the identity mapping $X \rightarrow X$ if for any point $x_{0} \in X$ and any neighborhood $V$ of $x_{0}$ there is another neighborhood $W$ of $x_{0}$ and an integer $N$ such that $x \in W$ and $n \geqq N$ imply $H^{n}(x, t) \in V$ for all $t$.

Recall that $A E\left(D, E^{2}\right)$ is the set $\left\{f \in A\left(D, E^{2}\right): f\right.$ is an embedding .

Lemma 11. $A E\left(D, E^{2}\right)$ is an $A N R$.

Proof. We shall use Theorem 10. Let $X=A E\left(D, E^{2}\right)$. For $i$ a positive integer, let $Y_{i}=\bigcap_{n=2}^{\infty} A\left(D, E^{2}, 1-1 / n\right)$, let $\phi_{i}: X \rightarrow Y_{i}$ be the inclusion map, let $\psi_{i}: Y_{i} \rightarrow X$ be defined by $\psi_{i}(f)(z)=f((1-1 / i) \cdot z)$, all $z \in D \subset \mathscr{C}^{1}$, and let $H^{i}: X \times I \rightarrow X$ be defined by $H^{i}(f, t)(z)=f((1-t) \cdot z+t \cdot(1-1 / i) \cdot z)$, all $z \in D$, $t \in I$. With these definitions it is easily checked that the hypotheses of Theorem 10 are satisfied, and so $X=A E\left(D, E^{2}\right)$ is an ANR.

Lemma 12. $E\left(\mathrm{Bd}(D), E^{2}\right)$ is an $A N R$. 
Proof. $E\left(\mathrm{Bd}(D), E^{2}\right)$ is the union of the space $E_{0}\left(\mathrm{Bd}(D), E^{2}\right)$ of orientation preserving embeddings of $\mathrm{Bd}(D)$ into $E^{2}$ and the space of orientation reversing embeddings of $\mathrm{Bd}(D)$ into $E^{2}$. Since these two spaces are homeomorphic and open in $E\left(\mathrm{Bd}(D), E^{2}\right)$, it suffices to show that $E_{0}\left(\mathrm{Bd}(D), E^{2}\right)$ is an ANR [12, Chapter 3, Theorem 8.1].

Given $f \in E_{0}\left(\operatorname{Bd}(D), E^{2}\right)$, choose an annulus $A(f)$ such that $f(\operatorname{Bd}(D))$ $\subset$ Int $(A(f))$, and $f(\mathrm{Bd}(D))$ separates Bd $(A(f))$. Define $x(f), w(A(f)), V(A(f))$ as in the paragraph preceding Lemma 2. By Corollary $4, w(A(f))$ is homeomorphic to $V(A(f)) \times \bar{H}(\mathrm{Bd}(D))$. It is clear that $\bar{H}(\mathrm{Bd}(D))$ is homeomorphic to the space $H(I)$ of orientation preserving homeomorphisms of the interval $[0,1]$ onto itself. But $H(I)$ is homeomorphic to $l_{2}$ [2], and thus is an ANR [12, Chapter 3, Corollary 6.4]. The space $Q=\left\{h \in A E\left(D, E^{2}\right): h(0)=x(f)\right\}$ is clearly a retract of $A E\left(D, E^{2}\right)$ and thus an ANR by Lemma 11. $V(A(f))$ is an open subset of $Q$, hence $V(A(f)$ ) is an ANR [12, Chapter 3, Proposition 7.9]. Therefore $w(A(f))$ being the product of two ANR's, is an ANR [12, Chapter 3, Proposition 7.6].

Finally, $w(A(f))$ is open in $E_{0}\left(\mathrm{Bd}(D), E^{2}\right)$, and

$$
E_{0}\left(\operatorname{Bd}(D), E^{2}\right)=\sum_{f \in E_{0}\left(\operatorname{Bd}\left(D, E^{2}\right)\right)} w(A(f)),
$$

hence $E_{0}\left(\mathrm{Bd}(D), E^{2}\right)$ is an $\mathrm{ANR}$ [12, Chapter 3, Theorem 8.1]. The proof of Lemma 12 is complete.

Lemma 13. $E\left(D, E^{2}\right)$ is an $A N R$.

Proof. As in Lemma 12 it suffices to show that $E_{0}\left(D, E^{2}\right)$ is an ANR.

Given $g \in E_{0}\left(D, E^{2}\right)$, define $g \mid \operatorname{Bd}(D)=g_{1} \in E_{0}\left(\operatorname{Bd}(D), E^{2}\right)$. For each $g \in E_{0}\left(D, E^{2}\right)$, let $A\left(g_{1}\right)$ be an annulus such that $g_{1}(\mathrm{Bd}(D)) \subset \mathrm{Int}\left(A\left(g_{1}\right)\right)$, and $g_{1}(\mathrm{Bd}(D))$ separates $\mathrm{Bd}\left(A\left(g_{1}\right)\right)$. Define $w\left(A\left(g_{1}\right)\right) \subset E_{0}\left(\mathrm{Bd}(D), E^{2}\right)$ as in the paragraph preceding Lemma 2. Define $T\left(A\left(g_{1}\right)\right)=\left\{h \in E_{0}\left(D, E^{2}\right): h_{1} \in w\left(A\left(g_{1}\right)\right)\right\}$. Then $T\left(A\left(g_{1}\right)\right)$ is open in $E_{0}\left(D, E^{2}\right)$, and $E_{0}\left(D, E^{2}\right)=\sum_{g \in E_{0}\left(D, E^{2}\right)} T\left(A\left(g_{1}\right)\right)$. It suffices, therefore, to show that $T\left(A\left(g_{1}\right)\right)$ is an ANR. Let $\sigma: w\left(A\left(g_{1}\right)\right) \rightarrow E_{0}\left(D, E^{2}\right)$ be the map of Lemma 3 such that $\sigma(f) \mid \operatorname{Bd}(D)=f$, all $f \in w\left(A\left(g_{1}\right)\right)$. Define $\gamma: T\left(A\left(g_{1}\right)\right) \rightarrow w\left(A\left(g_{1}\right)\right) \times H(D)$ by $\gamma(h)=\left(h_{1}, h^{-1} \circ \sigma\left(h_{1}\right)\right)$. It is easily checked that $\gamma$ is a homeomorphism. $w\left(A\left(g_{1}\right)\right)$ is an ANR by the proof of Lemma $12 ; H(D)$ is an ANR by [18]. Hence $T\left(A\left(g_{1}\right)\right)$ is an ANR and the proof of Lemma 13 is complete.

Suppose $Y$ is a spanning arc of the disk $D$. Then

LEMMA 14. $E(Y, D)$ is an $A N R$.

Proof. By Corollary 6, $E(Y, D) \times H\left(Z_{1}\right) \times H\left(Z_{2}\right)$ is homeomorphic to $\bar{H}\left(Z_{0}\right)$, where $Z_{0}, Z_{1}, Z_{2}$ are disks, $D \subset Z_{0}$, and $\bar{H}\left(Z_{0}\right)=\left\{h \in H\left(Z_{0}\right): h \mid Y \in E(Y, D)\right\}$. $H\left(Z_{0}\right)$ is an ANR by [18], so $\bar{H}\left(Z_{0}\right)$, being an open subset of $H\left(Z_{0}\right)$, is an ANR. But then $E(Y, D)$ is a retract of $\bar{H}\left(Z_{0}\right)$, hence $E(Y, D)$ is an ANR.

5. More on conformal mapping. In this section we describe a procedure for extending, in a canonical way, an embedding of the boundary of an annulus to an embedding of the entire annulus. This procedure is used in [10], [15], [19], and [22]. 
It is well known that if $G$ is a closed (topological) annulus in $\mathscr{C}^{1}$ then there is a unique real number $r>1$ and a homeomorphism $f$ of the annulus $A\left(C_{1}, C_{r}\right)$ $=\left\{z \in \mathscr{C}^{1}: 1 \leqq|z| \leqq r\right\}$ onto $G$ such that $f$ is holomorphic on Int $\left(A\left(C_{1}, C_{r}\right)\right)$. Further, $f$ is uniquely determined by the image of one boundary point $[1$, Chapter 5 , §3.1].

As in $\S 1$, we have a continuity property for such $f$ 's. In the statement below we let $A(J, L)$ denote the closed annulus bounded by the simple closed curves $J$ and $L$, with $J$ in the bounded complementary domain of $L$, and we let

$r$ a real number.

$$
C_{r}=\left\{z \in \mathscr{C}^{1}:|z|=r\right\},
$$

THEOREM 15. Given: (1) annulii $A\left(J_{n}, L_{n}\right), n=0,1,2, \ldots$, (2) homeomorphisms $f_{n}: A\left(C_{1}, C_{r_{n}}\right) \rightarrow A\left(J_{n}, L_{n}\right)$, with $f_{n}$ holomorphic on Int $\left(A\left(C_{1}, C_{r_{n}}\right)\right), n=0,1,2, \ldots$, (3) $\left\{J_{n}\right\} \rightarrow J_{0},\left\{L_{n}\right\} \rightarrow L_{0}$, 0-regularly, and (4) $\left\{f_{n}(1)\right\} \rightarrow f_{0}(1)$.

Then: (a) $\left\{r_{n}\right\} \rightarrow r_{0}$ (radii of outer boundaries of $A\left(C_{1}, C_{r_{n}}\right)$ converge), and (b) given a number $\varepsilon>0$, there is a number $\delta>0$ and an integer $N$ such that if, dist $(x, y)<\delta$, then dist $\left(f_{n}(x), f_{0}(y)\right)<\varepsilon$ whenever $n>N, x \in A\left(C_{1}, C_{r_{n}}\right), y \in A\left(C_{1}, C_{r_{0}}\right)$.

This theorem may be proved by methods similar to those in the proof of Lemma 12.1 in [21, Chapter 3, §12].

( $\star$ ) Definition. Let $A N$ denote the space of all orientation preserving embeddings $g$ of $C_{1}$ into Int $\left(A\left(C_{1 / 2}, C_{2}\right)\right)$ such that: (a) $g\left(C_{1}\right)$ separates $C_{1 / 2}$ and $C_{2}$, and (b) $g(1)$ lies in the interior of a small disk $O$, centered at 1 , so that the angle $\theta(g)$ at the origin from $g(1)$ to $2(=2+0 i)$ satisfies $-\pi / 4<\theta(g)<\pi / 4$.

Now if $g \in A N$ there is an annulus $A\left(C_{1}, C_{r}\right)$ and an embedding $G: A\left(C_{1}, C_{r}\right)$ $\rightarrow E^{2}$, holomorphic on Int $\left(A\left(C_{1}, C_{r}\right)\right)$, such that $G(1)=g(1), G\left(C_{1}\right)=g\left(C_{1}\right)$, and $G\left(C_{r}\right)=C_{2}$. If we precede $G$ with a radial homeomorphism $R$ taking $A\left(C_{1}, C_{2}\right)$ onto $A\left(C_{1}, C_{r}\right)$ and let $\lambda^{\prime}(g)=G \circ R$, then Theorem 15 gives us

LEMMA 16. There is a map $\lambda^{\prime}: A N \rightarrow E\left(A\left(C_{1}, C_{2}\right), E^{2}\right)$ such that $\lambda^{\prime}(g)(1)=g(1)$, $\lambda^{\prime}(g)\left(C_{1}\right)=g\left(C_{1}\right)$, and $\lambda^{\prime}(g)\left(C_{2}\right)=C_{2}$ for all $g \in A N$.

Suppose again that $g \in A N$. Let $g_{1}: C_{1} \rightarrow C_{1}$ be the homeomorphism $g_{1}=g^{-1} \circ \lambda^{\prime}(g) \mid C_{1}$. Let $g_{2}: C_{2} \rightarrow C_{2}$ be $g_{2}=\lambda^{\prime}(g) \mid C_{2}$. We may extend $g_{1}$ and $g_{2}$ to a homeomorphism $G(n, m)$ of $A\left(C_{1}, C_{2}\right)$ onto itself by sending $(r, \theta)$ (polar coordinates), to $\left(r,(2-r)\left(g_{1}(1, \theta)+2 n \pi\right)+(r-1)\left(g_{2}(2, \theta)+2 m \pi\right)\right)$ where $n$ and $m$ are integers. If we let $\lambda(g, n, m)=\lambda^{\prime}(g) \circ[G(n, m)]^{-1}$ we see that $\lambda(g, n, m)=g$ on $C_{1}$, and $\lambda(g, n, m)=\mathrm{Id}$ on $C_{2}$. Finally, let $\lambda(g)=\lambda(g, n(g), m(g))$, where the integers $n(g)$ and $m(g)$ are chosen as follows. If $X$ is the segment of the real axis from 1 to 2 , choose $n(g), m(g)$ so that the " angle change" along $\lambda(g, n(g), m(g))(X)$ is equal to the angle $\theta(g),-\pi / 4<\theta(g)<\pi / 4$, from $g(1)$ to 2 (see [10, p. 522] for a description of angle change); equivalently, choose $n(g), m(g)$ so that the "circulation index" (see [23, Chapter 5, §1]) of $\lambda(g, n(g), m(g)) \mid X$ about the origin has imaginary part $\theta(g)$. The continuity of $\lambda^{\prime}(g)$ and $\theta(g)$ as functions of $g$ imply the continuity of $\lambda$. Thus we have 
LEMMA 17. There is a map $\lambda: A N \rightarrow E\left(A\left(C_{1}, C_{2}\right), E^{2}\right)$ such that $\lambda(g) \mid C_{1}=g$, and $\lambda(g) \mid C_{2}=$ Id for all $g \in A N$.

For further discussion see [19].

6. $H\left(M^{2}\right)$ is an ANR. In this section we drop the superscript 2 and let $M$ denote a compact, metric, 2-manifold.

THEOREM 18. $H(M)$ is an $A N R$.

Proof. Since $H(M)$ is a topological group it is homogeneous. It is sufficient, therefore, to find an open subset of $H(M)$ which is an ANR and which contains the identity map [12, Chapter 3, Theorem 8.1].

As in [10] we proceed by induction on the number of cells in a cellular decomposition of $M$. Let $D_{1}, \ldots, D_{n}$ be a finite collection of disks such that (a) $M=\sum_{i=1}^{n} D_{i}$, (b) $D_{i} \cap D_{j}$ is either empty or an arc in $\operatorname{Bd}\left(D_{i}\right) \cap \operatorname{Bd}\left(D_{j}\right), i \neq j$, (c) $\mathrm{Bd}\left(D_{i}\right) \cap \mathrm{Bd}(M)$ is either empty, a simple closed curve, or a finite collection of arcs, and (d) each $D_{i}$ is the underlying point set of a subcomplex in a triangulation $T$ of $M$. Such a decomposition may be obtained, for example, by taking each $D_{i}$ to be the star, in the second barycentric subdivision of $T$, of the barycenter of a simplex of $T$.

If there is only one cell in the decomposition, then $H(M)=H\left(D_{1}\right)$ is an ANR by [18]. Assume that the theorem is true for manifolds which have a decomposition into fewer than $n$ elements. Let $\left\{D_{1}, \ldots D_{n}\right\}$ be a cellular decomposition of $M$ with $n$ elements. Let $D=D_{1}$.

Case 1. $D \cap \operatorname{Bd}(M)=\varnothing$. Let $N$ be a regular neighborhood of $\mathrm{Bd}(D)$ in $M$ (see [13, p. 57]), such that $N \cap \operatorname{Bd}(M)=\varnothing$. Then $D+N$ is a disk, hence $N$ is an annulus $\left(D+N=D+N^{\prime}\right.$, where $N^{\prime}$ is a regular neighborhood of $D$ in $M$, and $D+N^{\prime} \searrow D \searrow 0$, so $D+N^{\prime}$ is a 2-cell, see [13, p. 57]). We may think of $D+N$ as being embedded in $E^{2}$, with $N=A\left(C_{1 / 2}, C_{2}\right)$ and $\mathrm{Bd}(D)=C_{1}$ (unit circle). Let $A N$ be the subset of $E_{0}\left(C_{1}\right.$, Int $\left.\left(A\left(C_{1 / 2}, C_{2}\right)\right)\right)$, given in $\S 5$, Definition ( $\left.\star\right)$. Let

$$
H_{I}(M)=\{F \in H(M): F \mid \operatorname{Bd}(D) \in A N \text { and } F(D) \subset \operatorname{Int}(D+N)\} .
$$

Note that $H_{I}(M)$ is open in $H(M)$ and contains the identity map.

If we let $M^{\prime}=\sum_{i=2}^{n} D_{i}$, and let

$$
E_{I}(D, \text { Int }(D+N))=\{f \in E(D, \text { Int }(D+N)): f \mid \operatorname{Bd}(D) \in A N\},
$$

then $H_{I}(M)$ is homeomorphic to $H\left(M^{\prime}\right) \times E_{I}(D$, Int $(D+N))$. To see this let $\lambda: A N \rightarrow E\left(A\left(C_{1}, C_{2}\right), E^{2}\right)$ be the map of Lemma 17 such that $\lambda(f)\left|C_{1}=f, \lambda(f)\right| C_{2}$ $=$ Id, all $f \in A N$. Define $\lambda: A N \rightarrow E\left(M^{\prime}, M^{\prime}+N\right)$ by $\bar{\lambda}(f)=\lambda(f)$ on $M^{\prime} \cap N$, and $\lambda(f)=\mathrm{Id}$ on $M^{\prime}-N$. Then the map sending $F \in H_{I}(M)$ to $\left(F^{-1} \circ \bar{\lambda}(F \mid \operatorname{Bd}(D)), F \mid D\right)$ is the required homeomorphism. 
But $E_{I}(D, \operatorname{Int}(D+N))$ is an open subset of $E\left(D, E^{2}\right)$, and thus an ANR by Lemma 13. $H\left(M^{\prime}\right)$ is an ANR by our inductive hypothesis. Therefore $H_{l}(M)$ is an ANR, being the product of two ANR's.

Case 2. $D \cap \operatorname{Bd}(M) \neq \varnothing$. If $D \cap \mathrm{Bd}(M)$ is a simple closed curve, then $D$ is a component of $M$. Hence $H(M)=H(D) \times H\left(M^{\prime}\right)$, where $M^{\prime}=\sum_{i=2}^{n} D_{i}$, and $H(M)$ is an ANR by [18] and our inductive hypothesis.

Suppose, then, $D \cap \mathrm{Bd}(M)$ is a finite collection of arcs. Let $Y_{1}, \ldots, Y_{m}$ be the (disjoint) arcs making up the closure of $\mathrm{Bd}(D)-\mathrm{Bd}(M)$. For each $i, 1 \leqq i \leqq m$, we may choose a regular neighborhood $N_{i}$ of $Y_{i}$ in $M$ such that $N_{i} \cap N_{j}=\varnothing, i \neq j$, and $N_{i} \cap \mathrm{Bd}(M)$ is a regular neighborhood of $\mathrm{Bd}\left(Y_{i}\right)$ in $\mathrm{Bd}(M)$ and hence consists of two $\operatorname{arcs}$ in $\mathrm{Bd}(M)$ (see [13, p. 64]). Thus $N_{i}$ is a 2-cell (since $N_{i} \searrow Y_{i}$ $\searrow 0$ ) which meets $\mathrm{Bd}(M)$ in two disjoint arcs. Let $R_{i}$ be a smaller regular neighborhood of $Y_{i}$ such that $\mathrm{Bd}\left(R_{i}\right) \cap \operatorname{Bd}\left(N_{i}\right)=\operatorname{Bd}\left(R_{i}\right) \cap \operatorname{Bd}(M) \subset \operatorname{Int}\left(\operatorname{Bd}\left(N_{i}\right) \cap \operatorname{Bd}(M)\right)$.

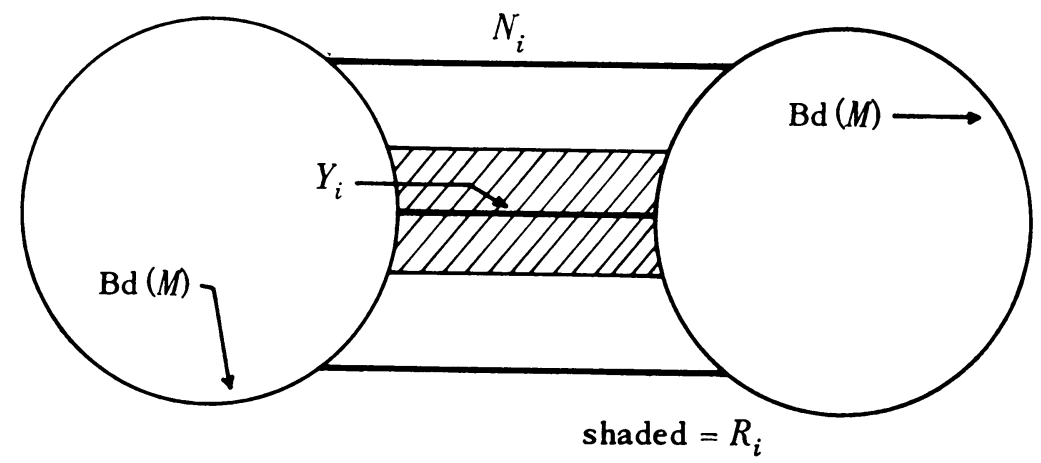

Let $E\left(Y_{i}, R_{i}\right), 1 \leqq i \leqq m$, be the space of embeddings of $Y_{i}$ into $R_{i}$ which are fixed on the endpoints of $Y_{i}$ and which take $Y_{i} \cap \operatorname{Int}\left(R_{i}\right)$ into Int $\left(R_{i}\right)$. By Lemma 5 there are maps $\gamma_{i}: E\left(Y_{i}, R_{i}\right) \rightarrow E\left(D \cap N_{i}, N_{i}\right)$ and $\alpha_{i}: E\left(Y_{i}, R_{i}\right) \rightarrow E\left(M^{\prime} \cap N_{i}, N_{i}\right)$, $M^{\prime}=\sum_{i=2}^{n} D_{i}$, such that $\gamma_{i}(f)=\alpha_{i}(f)=f$ on $Y_{i}, \gamma_{i}(f)=\operatorname{Id}$ on $D \cap \operatorname{Bd}\left(N_{i}\right)$, and $\alpha_{i}(f)=\operatorname{Id}$ on $M^{\prime} \cap \operatorname{Bd}\left(N_{i}\right)$, all $f \in E\left(Y_{i}, R_{i}\right)$.

Let $H_{I}(M)=\left\{F \in H(M): F \mid Y_{i} \in E\left(Y_{i}, R_{i}\right), 1 \leqq i \leqq m\right\}$. Note that $H_{I}(M)$ is open in $H(M)$ and contains the identity map.

Define $\gamma: H_{I}(M) \rightarrow E\left(D, D+\sum N_{i}\right)$ by $\gamma(F)=\gamma_{i}\left(F \mid Y_{i}\right)$ on $D \cap N_{i}, 1 \leqq i \leqq m$, and $\gamma(F)=$ Id on $D-\sum N_{i}$. Define $\alpha: H_{I}(M) \rightarrow E\left(M^{\prime}, M^{\prime}+\sum N_{i}\right)$ by $\alpha(F)=\alpha_{i}\left(F \mid Y_{i}\right)$ on $M^{\prime} \cap N_{i}, 1 \leqq i \leqq m$, and $\alpha(F)=$ Id on $M^{\prime}-\sum N_{i}$. But now the map sending $F \in H_{I}(M)$ to $\left(F\left|Y_{1}, \ldots, F\right| Y_{m}, F^{-1} \circ \gamma(F), F^{-1} \circ \alpha(F)\right)$ is a homeomorphism of $H_{I}(M)$ onto $E\left(Y_{1}, R_{1}\right) \times \cdots \times E\left(Y_{m}, R_{m}\right) \times H(D) \times H\left(M^{\prime}\right) . E\left(Y_{i}, R_{i}\right), 1 \leqq i \leqq m$, is an ANR by Lemma 14; $H(D)$ is an ANR by [18], and $H\left(M^{\prime}\right)$ is an ANR by our induction hypothesis. Therefore $H_{I}(M)$ is an ANR, and the proof of Theorem 18 is complete.

COROLlARY 19. If no component of $M$ is a 2-sphere, torus, projective plane, or Klein bottle, then the identity component of $H(M)$ is an absolute retract (and thus is contractible). 
Proof. The identity component of $H(M)$ is homotopically trivial by [9]. But every homotopically trivial connected ANR is an absolute retract [12, Corollary 8.5 , p. 219].

REMARK. In [25, p. 34] Earle and Eells remark that if $H_{0}(M)$, the identity component of $H(M)$, is an ANR, then the inclusion map of the identity component of the space of diffeomorphisms on (a suitably smooth) $M$ into $H_{0}(M)$ is a homotopy equivalence.

\section{BIBLIOGRAPHY}

1. L. V. Ahlfors, Complex analysis. An introduction to the theory of analytic functions of one complex variable, McGraw-Hill, New York, 1953. MR 14, 857.

2. R. D. Anderson, Spaces of homeomorphisms of finite graphs (to appear).

3. R. D. Anderson and R. H. Bing, A complete elementary proof that Hilbert space is homeomorphic to the countable infinite product of lines, Bull. Amer. Math. Soc. 74 (1968), 771-792. MR 37 \#5847.

4. A. V. Cernavskiř, Local contractibility of the homeomorphism group of a manifold, Dokl. Akad. Nauk SSSR 182 (1968), 510-513=Soviet Math. Dokl. 9 (1968), 1171-1174. MR 38 \#5241.

5. R. Courant, Dirichlet's principle, conformal mapping, and minimal surfaces, Interscience, New York, 1950. MR 12, 90.

6. J. Dugundji, Topology, Allyn and Bacon, Boston, Mass., 1966. MR 33 \#1824.

7. R. D. Edwards and R. C. Kirby, Deformations of spaces of imbeddings, Ann. of Math. 93 (1971), 63-88.

8. R. Geoghegan, On spaces of homeomorphisms, embeddings and functions, Trans. Amer. Math. Soc. (to appear).

9. M.-E. Hamstrom, Homotopy groups of the space of homeomorphisms on a 2-manifold, Illinois J. Math. 10 (1966), 563-573. MR 34 \#2014.

10. M.-E. Hamstrom and E. Dyer, Regular mappings and the space of homeomorphisms on a 2-manifold, Duke Math. J. 25 (1958), 521-531. MR 20 \#2695.

11. O. Hanner, Some theorems on absolute neighborhood retracts, Ark. Mat. 1 (1951), 389408. MR 13, 266.

12. S-T. Hu, Theory of retracts, Wayne State Univ. Press, Detroit, Mich., 1965. MR 31 \#6202.

13. J. Hudson, Piecewise linear topology, Benjamin, New York, 1969. MR 40 \#2094.

14. J. Keesling, Function spaces, flows, and Hilbert space, Proc. Conference on Monotone and Open Mappings, SUNY at Binghamton (to appear).

15. H. Kneser, Die Deformationssatze der einfach zusammenhangen Flächen, Math. Z. 25 (1926), 362-372.

16. G. S. McCarty, Jr., Homeotopy groups, Trans. Amer. Math. Soc. 106 (1963), 293-304. MR 26 \#3062.

17. W. K. Mason, The space $H(M)$ of homeomorphisms of a compact manifold onto itself is homeomorphic to $H(M)$ minus any o-compact set, Amer. J. Math. 92 (1970), 541-551.

18. - The space of all self-homeomorphisms of a 2-cell which fix the cell's boundary is an absolute retract, Trans. Amer. Math. Soc. 161 (1971), 185-205.

19. H. R. Morton, The space of homeomorphisms of a disc with $n$ holes, Illinois J. Math. 11 (1967), 40-48. MR 34 \#5066.

20. W. Rudin, Real and complex analysis, McGraw-Hill, New York, 1966. MR 35 \#1420. 
1972] SPACE OF HOMEOMORPHISMS ON A COMPACT TWO-MANIFOLD 285

21. W. Veech, A second course in complex analysis, Benjamin, New York, 1967. MR 36 \#3955.

22. N. Wagner, The space of retractions of the 2-sphere and the annulus, Trans. Amer. Math. Soc. 158 (1971), 317-329.

23. G. T. Whyburn, Topological analysis, 2nd rev. ed., Princeton Math. Series, no. 23, Princeton Univ. Press, Princeton, N. J., 1964. MR 29 \#2758.

24. - Problems on infinite-dimensional spaces and manifolds, Louisiana State University, Baton Rouge, La., 1969 (mimeographed).

25. C. Earle and J. Eells, A fibre bundle description of Teichmuller theory, J. Differential Geometry 3 (1969), 19-43.

26. M. Fort (Editor), Topology of 3-manifolds and related topics, (Proc. Univ. of Georgia Inst., 1961), Prentice-Hall, Englewood Cliffs, N. J., 1962. MR 25 \#4498.

Department of Mathematics, Rutgers University, New Brunswick, New Jersey 08903 